\title{
Spontaneous rupture of the middle colic artery resulting in delayed postpartum collapse: a case report
}

A.J Randawa MBBS, FWACS* drrandawa@yahoo.com, +234 8037019756, V Ajayi MBBS* victordayo@yahoo.com, +234 8034530698, S Albert MBBS* similalbert@yahoo.com, +234 8037871227

Department of Obstetrics \& Gyneacology, Ahmadu Bello University Teaching Hospital, Zaria, Nigeria*

Key words: Middle colic artery, rupture, postpartum collapse.

DOI:http://dx.doi.org/10.4314/ahs.v14i3.39

\section{Case presentation}

Rupture of the superior mesenteric artery branches is said to be a rare event, rupture of the middle colic artery being the most seldom. This report describes a 40 year old known hypertensive grandmultipara woman that presented with rupture of the middle colic artery in the immediate postpartum period.

A 40 year old known hypertensive grandmultipara woman presented eight days postpartum with a two days history of fever and a day history of abdominal pain. The abdominal pain was sudden in onset and initially on the right side but later became generalized. The pain was dull, severe and worsened by movement. There was no associated excessive vaginal bleeding. The lochia was normal and non malodorous.

She had delivered in the same hospital eight days prior to presentation via spontaneous vaginal delivery, though labour was augmented with oxytocin. She was on admission for three days after delivery due to raised blood pressure which was controlled using antihypertensives. The baby weighed $3.8 \mathrm{~kg}$ with good Apgar score.

On examination she was acutely ill, severely pale with a pulse rate of $126 / \mathrm{min}$ and a blood pressure of 110/80mmHg. The abdomen was distended, with generalized tenderness and guarding. There was shifting dullness on the right flank. Pelvic examination revealed normal lochia which was brownish. The cervix was short, closed but with marked cervical motion tenderness. The pouch of Doughlas was full.

\section{Correspondence:}

Dr A.J Randawa, drrandwa@yahoo.com

Department of Obstetrics and Gyneacology,

Ahmadu Bello University Teaching Hospital,

Zaria, Nigeria
Her packed cell volume was 19\% and abdominal ultrasonography showed an empty bulky uterus that measured $8.4 \mathrm{~cm}$ in maximum AP diameter. The myometrial echotexture was preserved. A presumptive diagnosis of silent uterine rupture with heamoperitoneum and or broad ligament heamatoma was made.

She was started on parenteral antibiotic and a pint of compatible blood preparatory to exploratory laparatomy. While being transfused she was noticed to be getting paller with decreasing blood pressure and increasing abdominal distension and pains.

Immediate resuscitative measures where put in place because she went into shock and subsequently had exploratory laparatomy under the preoperative diagnosis of intraperitoneal heamorrhage. At exploratory laparatomy the findings were those of massive heamoperitoneum of approximately 4 litres, massive heamatoma involving the whole of the transverse colon mesentery, normal liver, spleen, uterus tubes, ovaries and bladder. The uterus was about 14 weeks size and normal with no rent or broad ligament heamatoma seen. While effort was being made to perform hemicolectomy, patient had cardiac arrest 3 times and finally died after the third arrestdespite resuscitative efforts.

\section{Discussion}

Spontaneous intra-abdominal heamorrhage due to visceral artery is often called "Abdominal Apoplexy".1 Abdominal pain results from the mass effect of a rapidly expanding heamatoma on adjacent structure or from bowel ischeamia due to hypoperfusion. Mesenteric artery rupture resulting in heamatoma or massive intraperitoneal heamorrhage is said to be commonly due to aneurysms.(2) Aneurysms of the superior mesenteric artery branches are rarely reported, and colic artery aneurysms are not common. $(3,4)$ The common presenting symptoms are mild abdominal discomfort for several days prior to sudden of severe abdominal pain and 
swelling.

The diagnosis of middle colic artery aneurysm is difficult, because the aneurysms are small and usually asymptomatic until rupture(2). In this case the patient presented with abdominal pain and fever followed by massive intra-abdominal heamorrhage when resuscitative effort was instituted with blood transfusion and intravenous fluid therapy.

One important fallacy in this case was the fact that this patient presented in the early post partum period and because the mesenteric vessel rupture is a rare event, it was never thought of. Instead, an obstetric cause was all along entertained until when this was ruled out at laparatomy. The rupture was thought to result from an aneurysm of the middle colic artery because of the associated chronic hypertension in this patient which could possibly be associated with atherosclerosis.

The mortality following aneurysmal rupture has reported to be approximately $20 \%$ (5). The demise of this patient despite resuscitative efforts and timely intervention illustrate this fact in a resource constrained setting like ours. This case also illustrate an uncommon and clinically rare cause of postpartum collapse and maternal mortality for which vigilance is needed for early diagnosis and therefore timely intervention the catastrophe (maternal mortality) encountered in this case.

\section{References}

1.Stevensons IM, Mansfield AO, Temple JG; Abdominal Apoplexy. Br J Surg1978; 65:318-320 PubMed .

2. Osamu C, Hiroshi K, Makoto S, Soichiro Y, Hiroyuki K, Yasumasa K, Hiroyasu M. Spontaneous rupture of Dissecting Aneurysm of the Middle colic Artery. Tokai J Exp Clin Med. 2004; 29:4;155-158.

3. Rueter SR, Fry WJ, Bookstein JJ. Mesenteric artery branch aneurysm. Arch Surg 1968;93:498-499.

4.Verma BS, Bose AK, Bhatia HC, Katoch R. Superior mesenteric artery branch aneurysm diagnosed by ultrasound. Br J Radiol. 1991; 64:286-290 PubMed .

5. Stanley JC, Wakefield TW, Graham LM. Clinical importance and management of splanchnic artery aneurysms. J Vasc Surg. 1986; 3:836-840 PubMed . 